\title{
Le modèle Bassidji (Partie 3)
}

\section{Farhad Khosrokhavar}

\section{(2) OpenEdition}

1 Journals

\section{Édition électronique}

URL : http://journals.openedition.org/conflits/684

DOI : $10.4000 /$ conflits.684

ISSN : $1777-5345$

Éditeur :

CCLS - Centre d'études sur les conflits lilberté et sécurité, L'Harmattan

\section{Édition imprimée}

Date de publication : 15 octobre 1998

ISSN : 1157-996X

\section{Référence électronique}

Farhad Khosrokhavar, «Le modèle Bassidji (Partie 3) », Cultures \& Conflits [En ligne], 29-30 | automnehiver 1998, mis en ligne le 16 mars 2006, consulté le 30 mars 2021. URL : http://

journals.openedition.org/conflits/684; DOI : https://doi.org/10.4000/conflits.684

Ce document a été généré automatiquement le 30 mars 2021.

Creative Commons License 


\title{
Le modèle Bassidji (Partie 3)
}

\author{
Farhad Khosrokhavar
}

Le nouveau corps et son malaise

2 Chez les martyropathes le rapport entre le corps et le sang reproduit le rapport schizoïde entre le monde (où la révolution s'est enlisée) et la subjectivité désespérée du martyropathe. Le musulman authentique doit quitter le monde et séjourner hors de lui sur le même modèle que le sang qui doit quitter le corps et s'épancher au dehors. Le sang est au corps ce que l'ego est au monde: ils doivent se séparer, consommer leur rupture et se libérer réciproquement de cette impure contiguïté qui constitue l'essence même de la vie.

3 Pour le martyropathe, désespéré d'assister à la désagrégation de la révolution, le corps et le sang se comportent selon le modèle de la fission du mouvement révolutionnaire. Tout ce qui était maintenu en état de cohésion et d'unité fusionnelle à l'aube de la révolution, se dissocie et se désintègre; le rapport entre le corps et le sang, au début incarné, se distend, les éléments atomisés revêtant un sens désormais mortifère, s'intégrant parfaitement dans la religiosité nécrophile qu'ils portent en eux et que propage l'Etat en la personne même de Khomeyni.

4 Le corps martyropathe est habité par un intense sentiment de péché. Une relation ternaire s'instaure entre le corps, le sang et le péché : pour que le corps se purifie, il doit se libérer de son sang. De même, pour que le sujet puisse racheter ses péchés, il doit se purifier en se séparant d'avec le corps. Le sang, tant qu'il est dans le corps, l'alourdit, de même que le péché alourdit l'âme du martyropathe. Le salut ne vient que de l'allégement qui est, en réalité, un acte de dissociation : le corps évacue le sang, il s'en dissocie ; ce faisant, il perd de sa pesanteur pécheresse, il se fait pur en s'allégeant. Le fardeau du péché pour l'ego et le fardeau du sang pour le corps sont de même " nature ": le péché alourdit l'âme, le sang appesantit le corps. La guerre est en l'occurrence un prétexte pour se délester de ses péchés par la suppression sanglante de son corps. Pourquoi l'échappement du sang hors du corps est-il associé à la salvation du martyr? Parce que le sang, tant qu'il circule dans le corps, l'entretient et le revivifie, il est principe d'impureté. Mais sitôt qu'il s'échappe hors du corps, il le met à mort et dès lors, il se fait non pas principe de sa corruption, mais de sa purification. On ne peut se 
purifier que par la mort. De la sorte, la même substance, le sang, se voit revêtir deux sens diamétralement opposés selon qu'il s'associe à la vie ou à la mort. Dans le premier cas, il revêt une signification négative, dans le second, positive.

5 La conversion du sang en un principe mortifère s'exprime par l'utilisation des schèmes culturels du chiisme (le sang de l'imam Hoseyn, de sa famille, d'Abbas demi-frère de Hoseyn, ce dernier mort assoiffé et ensanglanté à Karbélâ...), réinterprétés et restructurés dans un sens mortifère. Dans un premier temps, le jeune révolutionnaire cherche à faire couler le sang de l'ennemi. Mais ce stade est dépassé à mesure que la révolution s'enlise, que l'espérance dans la réalisation de l'utopie s'amenuise et qu'une religiosité mortifère se substitue à celle du début optimiste de la révolution. La soif du sang est désormais inextinguible et sans objet spécifique. Elle cherche de manière indifférenciée soi-même ou autrui. Mourir ou faire mourir, tuer ou se faire tuer, attenter à ses jours ou à ceux de son prochain, s'anéantir et anéantir le monde dans le même mouvement apocalyptique, tout finit par s'équivaloir. L'identité devient totalement hétéronome, ce qui rend la tâche d'autant plus facile à l'Etat islamiste qui manipule avec aisance cette fraction de la jeunesse qui se trouve désormais à sa merci.

7 La vague du martyre est par ailleurs justiciable d'une analyse en termes de protomode : la volonté d'imiter l'autre dans la mort est un trait constant chez les jeunes dans le mouvement révolutionnaire. Le principe d'imitation renvoie à une société unifiée et homogénéisée où les hiérarchies traditionnelles à base d'identités différentielles et bien étanches ont largement disparu. On est face à une société en gestation douloureuse où la nouvelle homogénéité sociale s'exprime dans la capacité d'imitation des jeunes dans le martyre, c'est-à-dire là même où se constitue le nouvel " individu-dans-la-mort ".

Dans les sociétés en voie de modernisation, la mode suit une voie originale, se situant à mi-chemin des deux mondes de la tradition et de la post-modernité. Cette dernière s'introduit dans les relations sociales par le consumérisme des couches supérieures et moyennes, et dans les couches inférieures, de manière onirique, par les mass media.

Les figures de la mode sont sacralisées sous une forme semi-rigide, ni éphémères (comme dans les sociétés désenchantées) ni immuables ou perçues comme telle (comme dans les anciennes sociétés dominées par la tradition). Une des figures les plus sacralisées selon cette ligne de conduite en Iran post-révolutionnaire, est celle de Khomeyni. Par le passé, il aurait été un saint, ou bien le fondateur d'une secte ou d'un schisme. A présent, il se fait la figure du Sacré selon les lignes directrices de la " quasisainteté " (il devient imam, néologisme dans le nouveau sens que revêt l'expression dans son cas, renvoyant à sa sainteté sui generis comme dirigeant hiératique de la révolution). On reconnaît en lui un être qui procède de l'ici-bas et de l'au-delà, mais les relie sous une forme qui exclut la transcendance traditionnelle du Sacré (il s'adresse directement au " peuple ", il n'a pas le hiératisme des ulémas traditionnels). Son charisme est différent, dans son contenu mental, du mana du saint homme chiite traditionnel ${ }^{1}$. Il est semi-rigide dans sa sacralité, il est le mélange du saint et de l'homme de la loi, n'étant pas exclusivement un être sacré. Il est constitutif d'un univers en transition vers la modernité et procédant ainsi des catégories mentales où la mode, cette précarisation du Sacré, intervient comme dimension essentielle de l'affect juvénile. Il devient principe d'imitation, la distance étant incomparablement plus grande entre lui et ses jeunes imitateurs du point de vue de ces derniers, qu'entre les stars de la mode en Occident et leurs fans. 

mode " du martyre, après la révolution. L'exacerbation de la guerre et le désespoir des jeunes acteurs de la révolution induisent une véritable vogue du martyre. Celle-ci s'inscrit dans une conjoncture spécifique :

11

- la révolution a échoué dans l'accomplissement de ses visées mais les jeunes ne sont pas prêts à s'avouer cet échec; ils l'occultent, et ce désespoir mi-avoué, mi-occulté, donne lieu à la martyropathie: les martyropathes, eux, continuent à poursuivre l'utopie révolutionnaire d'une société islamique où prévaudrait la justice inflexible et l'unité des cœurs, au mépris d'une réalité où ces idéaux se défont au fil des jours. $\mathrm{Ne}$ pouvant les réaliser ici-bas, les martyropathes les réalisent dans leur mort. Ceci donne lieu à un principe d'individuation mortifère qui se généralise dans un mouvement collectif de désespoir qui s'accroît du fait même de son imitation par les uns et par les autres, le pouvoir déployant tout son attirail pour le mettre en scène, sous forme de cérémonies de deuil et de repentance, encourageant ainsi le phénomène de l'imitation par sa dramatisation.

12

- les couches populaires se dotent d'une " identité déshéritée " qui dégénère avec la désagrégation du mouvement en une identité hezbollah acrimonieuse et tyrannique.

13

- la jeunesse urbaine n'a aucun moyen concret par lequel affirmer son identité ; son énergie ne trouve aucun champ d'application dans la réalité. Elle l'exploite dès lors dans la destruction, l'autodestruction et la mort, encouragée en cela par l'Etat islamiste qui exploite son deuil de la modernité en le transformant en principe de réalisation de soi dans la mort.

14 Sur ce fond d'échec et de désastre que tout contribue à rendre " apocalyptique " pour les jeunes, un véritable habitus mortifère se fait jour qui a donné lieu durant quelques années à un entichement sans bornes pour le martyre. Dans ce contexte, la mode du martyre est un fait collectif où la participation à la mort se fait dans une ambiance spécifique d'émulation, d'imitation et de surenchère, qui rappellent les phénomènes de mode en général, et de mode vestimentaire, en particulier. Deux traits distinguent néanmoins ce phénomène: d'une part, il n'est pas directement vestimentaire, mais procède d'une sémiologie où c'est le corps qui est le support des signes mortifères, et non point l'habit. En second lieu, c'est une société en voie de modernisation qui s'y adonne et de ce fait, la mode du martyre n'a pas le non-sérieux voulu et le simulacre qui caractérisent ce phénomène dans les sociétés modernes. Bien au contraire, la participation se fait sur un mode " mortellement sérieux " et par l'occultation du simulacre. Cependant, l'aspect non-vestimentaire et somatique chez l'homme trouve son pendant vestimentaire chez la femme où la mode du voile trouve son champ d'application. La raison en est que le corps de la femme est dégradé par rapport à celui de l'homme. Le premier est spéculaire et le second, incarné. Le premier reflète, sur ses couvre-chefs, le désespoir des hommes et procède, ainsi, par procuration.

même que les phénomènes de mode ont pour fonction de permettre aux membres d'un groupe de se reconnaître et de constituer une identité propre, le jeune désespéré, lui, scelle son identité au sein de Bassidje, en martyropathe, privilégié par sa mort au service de l'islam. Il y a constitution d'une identité à part où on inverse un état subalterne (la jeunesse déshéritée, refoulée au bas de l'échelle sociale) en affirmation 
de soi en tant qu'être supérieur, pur, distinct des autres (identité martyropathe). On se différencie du reste de la société, vouée à l'impureté et on se dote d'une identité d'élite, en termes du pur. La mode du martyre accentue ainsi les traits distinctifs d'un groupe spécifique où les jeunes, en désespoir de se réaliser dans ce monde, entendent se constituer en " individus-dans-la-mort ", avec l'active contribution de l'Etat islamiste qui y puise son principe de légitimité tout au long de la guerre.

De même que dans la mode, le phénomène d'imitation joue un rôle prépondérant au sein de Bassidje, la volonté d'emboîter le pas aux martyropathes, de " poursuivre leur chemin ", est constamment présente tout aussi bien chez les martyrs ludiques. Comme dans les phénomènes de mode, l'aspect cérémoniel est de prime importance: on redonne à la vie cette dimension symbolique en voie de disparition en ville, chez les jeunes, victimes de la déstructuration. La jeunesse révolutionnaire, après la révolution et ses déboires, n'a plus aucune mission où investir son ardeur, si ce n'est dans le martyre, seule issue à un trop-plein d'énergie qui ne parvient pas à se canaliser ailleurs que dans la mort. Si la mode du martyre est désignée comme moyen de se distinguer, c'est parce qu'elle fait fond sur un profond désespoir collectif qui est le terroir où elle s'éclôt. Chez les martyrs ludiques, le jeu avec la mort se confond avec la joie de vivre dans une excitation collectivement partagée, où l'imitation des uns et des autres les poussent à la surenchère, mettant en jeu leur vie comme à la roulette russe.

Les avatars de la violence

La violence qui se dégage de la martyropathie n'est pas indissociable de son inscription dans Bassidje, même si, par ailleurs, le soubassement ultime de cette violence, à savoir le désespoir post-révolutionnaire, préexiste à cette organisation comme telle. Bassidje canalise ce désespoir dans le sens de la violence contre la société - par l'intimidation de ceux qui voudraient exprimer publiquement leur opposition à la tournure de la révolution et au pouvoir hezbollah et ce, par le recours aux jeunes révolutionnaires bassidji ou à l'Armée des Pasdaran, mais aussi, de la violence contre soi des jeunes martyropathes : ils se font violence dans leur rapport ambivalent à la révolution, où ils se culpabilisent de leur manquement à la révolution, manquement qui aurait été à l'origine de la faillite du mouvement révolutionnaire comme projet intra-mondain, c'est-à-dire, comme forme de réalisation de l'islam utopique dans ce monde.

La manière dont se déroule la violence est symptomatique. Pour les besoins de l'analyse, on peut distinguer trois moments qui, dans la réalité, sont très souvent imbriqués. Dans un premier temps, c'est le sentiment de la menace contre la révolution par l'Ennemi qui prévaut ; celui-ci est quasiment omniprésent et multiforme : ce sont les partisans des Etats-Unis, et plus généralement de l'Occident corrompu, les tenants de leur confort égoïste (les mostakbar, riches et arrogants), les musulmans nonrévolutionnaires qui refusent de se laisser enrôler sous la bannière de Khomeyni, etc. La liste peut se rallonger indéfiniment, à mesure que la faillite de la révolution rend nécessaire la lutte contre un ennemi paradoxal : d'une part, son omnipotence et son ubiquité rendent plausible la remise en cause de la révolution comme projet intramondain (se réalisant dans ce monde et pas dans l'au-delà ou dans la mort); d'autre part, plus cet ennemi se démultiplie et se diversifie et plus le révolutionnaire authentique doit renoncer aux idéaux révolutionnaires pour parer au plus pressé et sauver la révolution. A la limite, le sauvetage de la révolution passe par le renoncement aux idéaux révolutionnaires eux-mêmes. Mais arrive un moment où, à force de restreindre ses ambitions et de surseoir à la réalisation de l'utopie révolutionnaire, la 
révolution elle-même finit par ne plus avoir de vocation terrestre (toute réalisation concrète étant renvoyée aux calendes grecques). Dès lors, la mort devient le seul lieu de réalisation de soi, la mort qui est le comme le blanc laissé par la disparition progressive de tout ce qui donnait sens à la révolution. La mort devient le seul projet plausible dans une révolution qui n'a plus de projet et ceci rend le monde inhabitable pour le jeune martyropathe : ici-bas, désormais, tout est impur, le monde étant indigne du séjour du musulman authentique. Cette mort revêt d'autant plus un sens que le jeune martyropathe se sent de plus en plus coupable de l'avortement de la révolution. C'est lui qui a manqué de courage et d'abnégation, et, c'est pourquoi, par-delà les manigances de l'Ennemi, par-delà les coupables turpitudes d'une société incapable de s'adonner corps et âme à la révolution, c'est lui qui est responsable de la faillite de la révolution. La violence contre l'Ennemi prend alors pour cible l'être même de l'acteur révolutionnaire, et le sujet se transforme en martyropathe. Désormais, et c'est le troisième moment, autant le soi qu'autrui sont coupables et de ce fait, indignes de vivre. La lutte s'intensifie autant contre autrui que contre soi, et ceci, fonde la légitimité du projet d'annihilation collective comme forme prévalante de " purification de soi ", telle que la prône le martyropathe désespéré. Dans cette conjoncture apocalyptique, tout au long d'une guerre qui se prolonge au fil des ans (1980-88), la destruction de ce monde semble être la seule planche de salut pour le croyant authentique. La révolution n'a plus de vocation intra-mondaine, elle n'est plus de ce monde, pas dans le sens du retrait du martyropathe en lui-même à l'écart du monde, mais comme volonté de destruction s'étendant sur soi comme sur autrui, bref, la violence comme forme pure d'annihilation enveloppant indistinctement le soi comme autrui. La vie elle-même comme parcelle d'une totalité intra-mondaine est vouée aux gémonies. Ce qui prime, ce n'est plus la lutte contre l'Ennemi, mais contre la Vie ellemême, forme sournoise d'impureté. La violence qui se focalisait sur l'adversaire et le contre-révolutionnaire, prend pour cible le sujet lui-même, l'acteur révolutionnaire, et pour couronner le tout, le sujet et son prochain, amis et ennemis confondus. Le troisième moment est celui où l'hétéro-violence qui s'était un moment transformée en auto-violence (violence sur soi à cause du prétendu manquement du sujet à la révolution) se transforme en omni-violence, pouvant prendre pour cible autant le soi qu'autrui, l'un et l'autre étant des parcelles d'une existence intra-mondaine qui est à bannir au nom d'une pureté mortifère.

Les opportunistes et les ludiques

21 Le martyropathe est incontestablement minoritaire sur le plan numérique au sein de Bassidje, mais remplit un rôle symbolique majeur, autant dans la stratégie de légitimation du pouvoir qu'eu égard aux autres bassidji qui le perçoivent comme un être supérieur qu'ils ne peuvent pas égaler. Ceux-ci, rappelons-le, sont formés de deux autres groupes. D'une part, les opportunistes, ceux qui cherchent au sein de Bassidje le tremplin pour assurer leur ascension sociale dans une société où plus aucune opportunité n'existe pour les gens d'en bas pour assurer leur promotion; d'autre part, les ludiques, formés surtout d'adolescents qui entendent vivre intensément et à l'abri du besoin au sein de Bassidje, en mettant en jeu leur vie pour assurer en même temps leur transition à l'âge adulte. La dimension ludique manque cruellement dans la vie quotidienne au sein d'une société post-révolutionnaire où la vie est de plus en plus morose. Les ludiques s'accrochent à la guerre pour se divertir et avoir des sensations fortes. Dans les rangs de Bassidje, on devient libre par inversion de la hiérarchie d'âge : les jeunes commandent ou se font remarquer par leur intrépidité, ce qui leur donne un 
prestige supérieur à celui des aînés ; on fait preuve d'héroïsme en s'exposant à la mort, et on surmonte par ce biais l'adolescence et son infériorité. La guerre fait office de rite de passage, de l'adolescence à l'âge adulte, pour les adolescents bassidji qui abandonnent l'école et ses contraintes, la vie familiale en pleine pénurie pour une vie d'aventure et d'abondance relative au sein de Bassidje (on s'y nourrit bien, on est bien pourvu en vivres et couverts par une société à qui l'Etat extorque des fonds sous prétexte de bénévolat, pour venir en aide aux jeunes qui le soutiennent activement).

Le martyropathe meurt en désespoir de la vie, alors que le jeune adolescent de Bassidje accepte de mettre en danger ses jours par insouciance ludique, vivant intensément cette fête sanglante qu'est la guerre. Par ce biais, il ressuscite la fête de l'aube de la révolution et il transcende la vie morose de l'après-révolution pour se réjouir, oubliant ainsi la morosité ambiante dans une société que la joie a quitté, depuis que la guerre et la pénurie mettent à l'épreuve les rêves d'abondance révolutionnaire. C'est pourquoi, le martyr ludique entretient avec la mort une relation totalement différente de celle du martyropathe. Le jeune ludique, en plein champ de bataille, refuse l'institutionnalisation de l'utopie révolutionnaire. Le sacré est pour lui dans l'effervescent, le bouillonnant, le fusionnel, affect qu'il oppose à la logique froide des richissimes commerçants du bazar ou aux calculs mesquins et prosaïques de ses parents qui ne savent trop comment joindre les deux bouts dans la vie quotidienne. Toute stabilisation est pour lui synonyme de la remise en cause de l'islam utopique qu'il porte en lui et entraîne, de sa part, la surenchère dans le combat. La guerre joue pour cette jeunesse ludique, le rôle d'opérateur de désinstitutionnalisation dans l'effervescence du champ de bataille. La mort ne procède pas d'un sombre désespoir, mais du désir d'extase et d'effervescence, de l'aspiration à remettre en cause la monotonie et l'ennui mortel d'une vie post-révolutionnaire où les déshérités sont encore plus démunis, encore plus vulnérables et par conséquent plus frustrés dans leur consommation que par le passé. Cette mort n'a pas le sombre caractère de la martyropathie. Elle est refus d'institutionnalisation, et non pas volonté de mourir à tout prix.

L'aspiration à vivre intensément des adolescents est récupérée par l'Etat islamiste par le truchement de Bassidje. Il forge un nouveau lien politique à partir de ce sentiment, en se légitimant et en marginalisant l'opposition politique qu'il refoule par le spectacle de la dévotion des jeunes : s'ils acceptent de mourir pour le credo islamiste, c'est que l'ordre khomeyniste est légitime. La manipulation des désirs d'une jeunesse immature, avide de jouissance, donne un poids considérable à l'ordre islamiste dont la légitimité est pourtant bâtie sur une vision néo-puritaine des relations sociales et culturelles.

Les opportunistes instrumentalisent l'islam du Hezbollah pour joindre les classes aisées $\mathrm{du}$ nouveau régime. Ils forment une nouvelle élite qui, moyennant les nouveaux réseaux révolutionnaires tissés par l'Etat islamiste, se substitue aux anciennes classes dominantes et moyennant sa fidélité vénale au régime, s'assure une vie à l'abri du besoin. Les institutions qui ont vu le jour pendant ou après la révolution leur servent de tremplin: la Croisade pour la Construction qui monopolise les projets du développement rural (plus tard elle se transformera en ministère), la Fondation des Martyrs qui est un colosse économique ayant ses propres moyens financiers et industriels, l'Astané Ghods qui gère les biens de l'imam Réza au nord-est de l'Iran, et qui est l'une des institutions les plus dotées de l'Iran², les divers comités révolutionnaires (ultérieurement intégrés dans le ministère de l'intérieur), l'Armée des Pasdaran, etc. Toutes ces nouvelles organisations révolutionnaires deviennent le 
moyen d'ascension sociale et économique pour une jeunesse avide de consommation et qui trouve, par son affiliation intéressée à Bassidje, et moyennant son exposition au danger, la possibilité de s'introduire dans l'appareil d'Etat et de bénéficier des mannes pétrolières. Une partie de la plèbe urbaine y trouve son compte, notamment par le truchement des Comités et de l'Armée des Pasdaran, intimidant dans les quartiers, mais intervenant le cas échéant pour soutenir leurs amis et leurs connaissances au sein de ces quartiers, auprès des instances répressives du régime où ils ont leurs entrées. Une nouvelle forme de clientélisme avec l'Etat se constitue avec cette jeunesse populaire qui monnaye sa collaboration au régime, en s'assurant, au niveau même du quartier, une nouvelle forme d'honorabilité, faite de la crainte des autres, mais aussi de leur respect, en raison de la capacité non négligeable d'aide que peut leur apporter cette jeunesse en ascension, qui a su s'introduire à temps dans les réseaux répressifs du régime. Par leur biais, la répression devient plus poreuse dans les quartiers populaires, en raison notamment de cette corruption rampante qui arrondit les angles aigus des choses, le système devenant moins redoutable et moins répressif, à mesure qu'il devient plus corrompu, notamment par le biais de cette jeunesse opportuniste qui cherche à promouvoir sa cause en épousant, de manière intéressée, la cause du Hezbollah.

La désintégration du modèle bassidji

Deux faits majeures préludent à la désintégration du modèle bassidji. D'une part, la fin de la guerre dérobe à la fraction martyropathe la capacité de se faire mourir en martyr et d'entretenir la confusion entre sa volonté de suicide et sa transcription selon un registre sacré ; d'autre part, la mort de Khomeyni, peu après la fin de la guerre (4 juin 1989), ôte aux derniers groupes martyropathes la possibilité de se réclamer d'un chef charismatique pour donner, par leur mort, une version tragique de la faillite de la révolution.

Par ailleurs, du fait même de la longue durée de la guerre (1980-88), l'ardeur martyropathe a eu tout le loisir de s'exercer, de se répandre par mimétisme selon un modèle assimilable à celui de la mode, et de s'épuiser progressivement au contact d'une réalité inhospitalière vis-à-vis de l'utopie révolutionnaire. Passées les premières années où le désespoir révolutionnaire se transcrivait aisément dans le registre de la mort sacrée (le martyre), petit à petit le réel a repris le dessus et la révolution s'est " prosaïsée " au contact de l'économie parallèle, du marché noir, de la vie quotidienne où l'abnégation révolutionnaire s'attiédit inexorablement, et de l'usure de la nouvelle élite qui se révèle aussi vénale et aussi peu " proche du peuple " que celle du temps du chah. $\mathrm{Au}$ début, la jeunesse révolutionnaire s'insurgeait contre le réalisme de ses parents et au nom de la préservation de l'islam révolutionnaire, elle rejoignait Bassidje. Peu à peu, la vie reprend le dessus et l'enthousiasme de la mort sacrée se transforme en un détachement cynique qui s'exerce dans la vie quotidienne, lieu par excellence de l'apprentissage de la dualité vis-à-vis du nouveau pouvoir : à son idéologie légitimante de sacrifice et d'abnégation s'oppose désormais l'égoïsme forcené de " l'individu ensauvagé " qui ne connaît de légitimes que ses intérêts bruts et qui instrumentalise les mots d'ordre révolutionnaires, de plus en plus vidés de leur substance, en adjuvant de ses intérêts " bassement matériels ". Ainsi en est-il de la demi-barbe révolutionnaire (comme un visage mal rasé que les jeunes hezbollahs affublaient dans les premières années pour se démarquer autant de la barbe piétiste traditionnel, longue et drue, que du visage glabre de l'occidentalisé); désormais, on l'affiche pour se donner un air de dévotion, tout en pratiquant des formes plus ou moins déviantes de spéculation sur un 
peu toutes les denrées (dollar, or, viande surgelé, produits électroménagers, etc.). De même, ceux qui sortent de Bassidje monnayent leur appartenance à cette organisation pour trouver des sinécures, notamment en matière d'approvisionnement des denrées dans une société où s'est installée la pénurie et où la différence entre les prix officiels et les prix du marché libre sont importants (un entrepreneur soutenu par Bassidje ou par l'une des nombreuses organisations révolutionnaires se trouve en situation d'avantage écrasant par rapport à ses rivaux du marché). De plus en plus, ceux qui y parviennent, monnayent leur appartenance à Bassidje, à l'Armée des Pasdaran, à la Croisade pour la Construction... en termes économiques, faussant ainsi les lois du marché et créant, de facto, un double marché qui défavorise les initiatives privées des plus démunis en ressources relationnelles. Ce néo-clientélisme où l'Etat post-révolutionnaire est instrumentalisé engendre sur le long terme une grande frustration de la part de la grande majorité de la jeunesse bassidji qui n'a pas pu transcrire la logique de son abnégation en participation intéressée aux ressorts de l'économie. Marginalisée, réduite à des formes de plus en plus patentes d'exclusion économique, cette jeunesse là, abandonnée en majorité à son sort par Bassidje à la fin de la guerre, forme la base d'un mécontentement brut qui s'exprime, au fil des années, par des mouvements de révolte spontanéiste et sporadique que le pouvoir parvient à mater sans difficulté, moyennant la répression ${ }^{3}$. Ce faisant, sa légitimité s'use d'autant, auprès de ceux-là mêmes qui avaient été le fer de lance de la révolution et de la répression de l'opposition, dont une partie se trouve refoulée dans l'opposition et rejoint les groupes plus ou moins radicaux du Hezbollah (par rapport aux " réalistes " qui se réclament de l'ouverture timide de l'Iran vers le monde extérieur).

Nombre de ceux qui avaient combattu au sein de Bassidje ou de l'Armée des Pasdaran rejoignent l'Etat et, que ce soit dans l'administration ou dans les structures industrielles, ou dans le secteur privé, ils constituent une frange de la nouvelle élite post-révolutionnaire qui finit par perdre sa conviction d'antan pour lui substituer une logique d'intérêt personnel sous la bannière d'un Etat qu'ils privatisent à leur manière. Peu d'idéaux révolutionnaires ont pu résister à l'usure du temps : la liberté (une forme de liberté sous surveillance, pourvue de certaines marges de manœuvre, se constitue progressivement après la mort de Khomeyni chez les intellectuels " post-islamistes ", en Iran même), l'indépendance économique (l'Iran est toujours très largement tributaire du pétrole à tous les niveaux de son économie), la pureté islamique (le privé est le lieu de transgression de tous les interdits que l'on respecte dans l'espace public non sans de graves entorses, dès que la vigilance du pouvoir se relâche momentanément), la vocation mondialiste de la révolution islamique à s'exporter (sauf au Liban où s'est constitué une milice hezbollah pro-iranienne, partout ailleurs le succès a été mitigé). Il ne reste plus que la répression des femmes (elles servent de bouc émissaire) et des formes ostentatoires, de plus en plus vidées de sens, de manifestations et de défilés, largement pris en charge par la nouvelle clientèle du pouvoir, pour affirmer les vertus salvifiques d'un islam dont la forme militante régresse au niveau de la société. Sur les ruines du mouvement islamiste, et au sein d'une société dominée par un pouvoir islamiste, se constitue, non sans difficultés, le noyau futur d'une société civile par le truchement d'intellectuels qui ne raisonnent plus ni en termes de révolution du prolétariat, ni en termes de révolution islamique ${ }^{4}$.

29 Ainsi, la désintégration $\mathrm{du}$ modèle bassidji s'effectue de deux manières complémentaires. D'une part, par l'intégration d'une partie des jeunes bassidji dans l'appareil d'Etat aux échelons inférieurs et moyens, que ce soit dans le secteur militaire 
(dans l'Armée des Pasdaran) ou civil (organismes de surveillance de l'administration ou dans les secrétariats où les femmes sont de plus en plus remplacées par les hommes) ou dans les organismes révolutionnaires qui se transforment progressivement en ministères et qui ont besoin de recrutement du personnel (comme la Croisade pour la Construction). D'autre part, par l'abandon à leur sort d'une grande partie des jeunes bassidji, chez qui s'accumulent la frustration et qui la manifestent occasionnellement, de manière éclatée, par des révoltes épisodiques dans les villes. Au sentiment, jadis entretenu, de la mort sacrée pour l'islam se substitue désormais celui de la révolte désespérée contre un pouvoir qui n'est islamique que de nom et qui usurpe le nom d'islam aux fins de promouvoir la fin de l'islam révolutionnaire.

31 La crise du mouvement révolutionnaire dans les premières années, puis son effondrement dans les années suivantes emportent les dernières illusions du martyropathe sur l'améliorabilité du monde. Il ne parvient plus à croire à l'avenir. L'échec de la révolution a révélé la dérision de la révolte dans le monde. Le sacré qui devait se réaliser dans le monde, sur le plan politique, se dissocie désormais du monde. Il s'agit de s'insurger contre le monde in toto (le soi en chair et en os étant inclus dans ce monde à abattre), de mettre fin à une existence rongée par la perte du sens. La mort, dans la conjoncture post-révolutionnaire, est une panacée pour les jeunes pris au dépourvu devant la débâcle de la révolution. L'impératif révolutionnaire de changer le monde se métamorphose en celui de changer de monde et ce, en s'expatriant de celui qui a occasionné l'écroulement du mouvement révolutionnaire. Pendant la révolution, la violence s'exerçait contre le régime impérial, principe du Mal. Après la révolution, le principe du Mal n'est plus aisément identifiable : l'optimisme ingénu de la révolution se change en un pessimisme angoissé. La violence se détourne du monde (il n'est plus le lieu du sens mais du non-sens et à ce titre, à détruire) pour se fixer sur le nouvel individu. Mais désormais la mort peut tout aussi bien s'asséner aux autres comme parcelles d'un monde à détruire. Le terrorisme du jeune martyropathe est avant tout un auto-terrorisme (il veut se mettre à mort) mais son registre est tel qu'il embrasse tout aussi bien, et indifféremment, les autres et dès lors, se transforme aisément en hétéro-terrorisme. Mourir ou faire mourir, le soi et les autres, dans un acte qui devient la dernière manifestation de soi au sein d'une communauté fictive (les " frères " de Bassidje, avec la bénédiction de Khomeyni qui sacralise la mort) revêt alors un sens existentiel. En s'assénant la mort, le martyropathe se venge du monde à travers soi, il jette l'anathème sur le monde qui engendre ce sentiment absurde de l'existence, le brise en anéantissant son propre corps. Le martyropathe croit se battre contre le monde, mais en réalité, il ne fait que se détruire. La martyropathie ne s'articule pas sur un projet positif de vie, elle est pure négativité, l'annihilation de soi par incapacité de s'assumer. Mais c'est aussi une affirmation de soi qui ne se réalise que dans le suicide. Elle est régressive et indice d'un soi désormais incoercible. Elle témoigne de la nonmaitrise du conflit existentiel qui surgit dans toute modernisation, mais aussi de la fin de l'ère communautaire où le sujet autonome n'existait pas. Le martyropathe est un " individu hétéronome " et non pas l'expression individualisée d'un ordre communautaire et traditionnel. C'est pourquoi, la logique de ses aspirations n'est pas une chose provisoire. Son désir d'être s'étanche dans la mort, mais comme désir, il est là et on ne saurait l'ignorer. Il est déjà du côté de la modernité dans la terreur qu'il répand et dans le suicide où il prétend se réaliser. 

endoctrine le jeune Bassidji, alors que dans le cas du jeune Palestinien, ce sont des groupuscules radicalisés. Celui-ci, du moins dans les années quatre-vingt, semblait provenir de milieux fragilisés, au sein de familles déstabilisées 5 . Dans les années quatrevingt-dix, surtout avec la dégradation des conditions socio-économiques, cette forme de " vocation " tend à se généraliser paradoxalement, du moins pour un temps, lorsque la perspective de la paix avec Israël devient plausible sous les travaillistes : la hargne et le désespoir contre un pays dont l'armée d'occupation a massivement réprimé, dans des relations de face à face quotidienne, la jeunesse palestinienne, pendant de nombreuses années, a engendré des envies d'en découdre avec l'adversaire qu'on refuse de percevoir sous la figure de voisin neutre avec qui on entrerait dans des rapports de voisinage non-antagonique. A la violence qui a sévi pendant longtemps, une partie de la jeunesse palestinienne répond par la volonté de rupture, pour prévenir une paix synonyme d'occultation de la violence qu'elle a subie. En d'autres termes, la paix, avant l'avènement de la droite israélienne au pouvoir, signifiait la mise sous le boisseau de la souffrance endurée. Elle était sensée mettre fin à la violence sans pour autant reconnaître la violence subie par les jeunes palestiniens. Ce malaise se transforme en désespoir total (et en cela on retrouve une des modalités du désespoir, qui, dans le cas iranien est lié à l'échec de l'utopie révolutionnaire), et les groupes radicaux qui s'en emparent, l'exploitent, pour endoctriner le jeune Palestinien en sacralisant ce désespoir. Les modalités de l'exploitation du désespoir portent quelque ressemblance avec les formes d'intervention de Bassidje: on tente de séparer mentalement (et physiquement, si possible), le jeune de sa famille : désormais, on lui construit un schéma d'accès au paradis où il se promeut individu-dans-la-mort, à l'écart des parents, des amis et des connaissances. La mort de soi devient alors le martyre qu'on relie à la Djihad (la guerre sainte) selon une modalité qui ne présente pas de différence majeure, sur le plan doctrinal, avec le chiisme, à ceci près qu'ici, on ne prend pas comme parangon la figure paradigmatique de l'imam Hoseyn ${ }^{6}$.

Ainsi, de nombreux traits qui se retrouvent, dans un cas comme dans l'autre, se fondent-ils sur un désespoir spécifique, celui de ne pouvoir se construire dans ce monde comme être s'épanouissant selon les aspirations instillées par une vie en voie de modernisation au sein d'une société en crise. Ce désespoir est d'autant plus aisément susceptible de se sacraliser qu'il met fin, par le martyre, à une vie devenue insupportable, sans projet, sans avenir, sans la moindre ombre de réalisation de soi. Ce n'est pas la spécificité chiite, liée à la sotériologie chiite (le Douzième Imam occulté7) et à son dolorisme (les célébrations doloristes du martyre de l'imam Hoseyn à Karbéla), qui donne sens au martyre, mais une quotidienneté vécue comme profondément inacceptable, la vie elle-même devenant dérisoire et la mort seule revêtant un sens. Les jeunes d'origine sunnite peuvent épouser cette vision du martyre sans renoncer pour autant à leur appartenance au sunnisme et sans ressentir pour cela une violence contre leur religiosité musulmane " orthodoxe ". Le martyre chez les bassidji tout comme chez certains jeunes Palestiniens ou certains chiites libanais est en réalité un néo-martyre: c'est la figure de l'individu-dans-la-mort qui ne parvient pas à se réaliser dans ce monde et qui, de ce fait, accepte d'aller jusqu'au bout, la facilité avec laquelle il met fin 
à ses jours dénotant un nouveau rapport entre la vie et la mort sur la base même de la modernisation mentale des jeunes. Ceux-ci savent désormais chérir davantage leurs rêves et leurs aspirations que la vie elle-même. Le néo-martyre qui se développe est d'autant plus intense que l'on vit douloureusement l'effondrement de soi au sein d'une société où tout changement positif est vécu comme impossible (du fait de l'occupation israélienne pour les jeunes Palestiniens, ou de la guerre irakienne pour les jeunes bassidji). Les jeunes bricolent alors une légitimation religieuse, non sans puiser dans l'arsenal théologique plus ou moins fruste dont ils disposent. Il est vrai que le chiisme, par le modèle paradigmatique de l'imam Hoseyn et de nombreux autres saints martyrs offre une remarquable facilité par rapport au sunnisme. Mais, les jeunes sunnites n'hésitent pas, à leur tour, à emprunter à foison au chiisme, la justification étant faite par des groupes où œuvrent des théologiens radicalisés.

C'est dans une modernité avortée (ou ressentie telle) et dans le processus d'individualisation " sauvage " des jeunes, de plus en plus désinsérés de leur communauté traditionnelle, et s'intégrant plus ou moins volontairement à des néocommunautés " déviantes " (groupuscules révolutionnaires ou groupes " eschatologiques " ou ensembles plus ou moins hermétiques) qu'il faut chercher la vague de fond de ce type de martyre. Celui-ci est, sous des apparences traditionnelles, tout sauf la reproduction de la tradition: il ressort d'un néo-martyre, la logique d'affirmation de soi dans la mort y prenant une place prépondérante (alors que la vie n'est plus perçue comme un lieu d'auto-affirmation pour l'ego). De même, ce " quasiindividu ", fraîchement dégagé des attaches communautaires, est fragile, il peut aisément adhérer à des formes néo-communautaires où son volontariat peut donner lieu à une manipulation et à des formes de servitudes beaucoup plus accentuées que dans les communautés traditionnelles, froides, hiérarchiques et négatrices de l'individualité. Ici, dans ces néo-communautés, le sujet vit in vitro soit la fin des temps, soit son annonce, soit la sacralisation en instance de mourir, soit une forme de fraternisation qui n'existe plus dans la société extérieure, lieu d'atomisation, d'égoïsme forcené et d'impossible épanouissement de soi.

Dans ces cas précis, on a un individu en instance de formation et qui ne trouve pas dans la société de lieu où il puisse s'épanouir. Qu'à cela on ajoute une logique d'humiliation constante : pour ce qui est de l'Iran, les Etats-Unis et leur armée, 40000 hommes, régis par des lois de Capitulation de type colonial - l'extraterritorialité juridique alors même qu'ils stationnaient en Iran ; pour ce qui est des jeunes Palestiniens, le déni d'existence dans la dignité, dans des territoires où ils sont niés dans leur être par l'armée israélienne et, sous une autre forme, incomparablement moins avilissante mais néanmoins insupportable, par leurs propres aînés (l'Intifada n'était pas le fait des adultes et des parents, mais des jeunes, contre l'armée israélienne, mais aussi, et indirectement, contre les parents qui n'avaient fait que " palabrer ").

La religiosité des martyropathes iraniens rejoint celle des jeunes radicalisés au sein du Hamas ou du Djihad, dans la mesure où leur affect se construit sur le fond d'échec du nationalisme comme idéologie légitimante de la modernisation. Dans le cas iranien, cet échec est beaucoup plus mental et politique qu'économique ${ }^{8}$. Dans le cas palestinien, on assiste à l'impossibilité de se doter d'une " nation ", et les formes laïques du nationalisme montrent rapidement leurs limites, face à une logique régionale et internationale qui refuse dramatiquement l'accès à la nation aux jeunes Palestiniens. De même, la présence écrasante de l'armée israélienne dans les territoires occupés 
donne une cible bien précise à la haine des jeunes Palestiniens, alors que les reproches des Iraniens sur la présence américaine en Iran et la constitution de l'Iran en une " sous-Amérique " régionale pour faire avancer les visées des Etats-Unis dans ses ambitions géostratégiques, tout en marquant un profond ressentiment, sont sans commune mesure, avec le sentiment de rejet qu'éprouvent les jeunes Palestiniens vis-àvis de l'armée israélienne.

Mais, par-delà ces phénomènes, les mêmes formes extrêmes de désespoir montrent une affinité élective avec le religieux : au-delà d'un certain seuil, lorsque le désespoir n'est plus maîtrisable par le sujet, il a tendance à se constituer en " Sacré mortifère " pour des êtres qui ne trouvent plus de consolation ni de bornes dans les communautés traditionnelles, irréparablement déstructurées par une " modernisation négative ". Celle-ci désarticule les anciennes formes de sociabilité sans avoir le dynamisme nécessaire pour leur en substituer de nouvelles. D'un côté, un individu qui émerge en filigrane à partir de la désarticulation des anciennes formes communautaires; de l'autre, aucune intégration positive au sein de la société; enfin, une existence quotidienne qui instille le désespoir et rend la vie inattrayante et révoltante. L'ensemble est propice à la constitution d'un désespoir sacralisé qui se nourrit du religieux déstructuré pour constituer l'individu-dans-la-mort. La sécularisation du religieux (on n'est pas dans des sociétés totalement laïques) rend possible cette forme d'expression de soi où le sujet renoue avec le sacré en se vouant à la mort et, dans le même acte, en brisant ce qui est vécu par lui comme à l'origine de son désespoir, que ce soit les Israéliens indistinctement mis à mort dans un bus par un jeune bardé d'explosif, ou les soldats irakiens que ne craint pas d'affronter le martyropathe dans sa furie de rompre avec la vie.

Dans cette situation, le vecteur religieux change de sens : alors qu'il est dans la grande majorité des cas, le lieu même de l'éclosion de l'espérance, à présent, le sacré rend possible le passage de la vie au trépas selon une volonté de rupture qui y trouve la possibilité de se déployer en corroborant cette volonté. Le religieux rend ainsi possible la sacralisation du désespoir. En ce sens, l'idéologie religieuse s'est substituée aux idéologies laïques qui prônaient la victoire finale par le dépassement de l'individu dans le grand creuset révolutionnaire dans les années soixante et soixante-dix.

Cependant, la différence essentielle qui sépare la forme laïque de l'activisme révolutionnaire des années soixante-dix de celle de la vision activiste du religieux dans les années quatre-vingt et quatre-vingt dix est, du moins dans le cas iranien, le désespoir intense causé par l'avortement inavoué du projet révolutionnaire, dans le cas palestinien, le désespoir de se doter d'une identité nationale au sein d'une société où il puisse concilier ses rêves de modernité et la réalité quotidienne dans l'indépendance nationale. Cette dissociation entre la logique d'une subjectivité en voie de modernisation et une réalité de plus en plus régressive est susceptible de se dénouer au sein d'une idéologie " religieuse " dans la mesure même où le sujet est capable de percevoir une totalité religieuse comme fondement du sens. Pour cela, il est nécessaire que la sécularisation n'ait pas été complète, que le sujet se trouve dans un entre-deux : entre d'une part une logique d'affirmation de soi individuelle (la sortie des communautés traditionnelles qui se déstructurent et qui ne sont plus capables d'intégrer les jeunes en leur sein), d'autre part, une vision de soi et du monde couchée en termes religieux. Le sens se construit non pas en rupture avec l'univers religieux (ce qui n'aurait pas de sens pour le jeune déshérité iranien et le jeune Palestinien à 
l'abandon) mais en son sein, moyennant la radicalisation de ses thématiques, voire la conversion de certains thèmes en incitation à l'action radicale, à l'action mortifère.

Un autre trait de cette forme de religiosité est sa fascination pour la mort. La rupture avec les communautés traditionnelles brise le rapport à la mort comme une transcendance. Le jeune acteur ne perçoit plus sa vie comme une fatalité, il prend son destin en main et ce faisant, son rapport à la mort se trouve profondément bouleversé. Alors que ses parents percevaient la vie comme une transcendance au même titre que le Sacré, qu'attenter à la vie leur paraissait profanatoire, à présent, les jeunes nouent un rapport à la vie et à la mort qui les rend immanentes l'une et l'autre. Dès lors, ils peuvent aller jusqu'à se défaire de leur vie, celle-ci n'étant plus du ressort de l'intangible, mais pouvant parfaitement s'instrumentaliser dans un mode d'être où on peut passer outre son existence, au nom même de sa subjectivité. C'est parce que le jeune bassidji iranien ou le jeune Palestinien ne sont plus liés à la tradition qu'ils peuvent affronter l'épreuve de la mort sous une forme radicalisée, mourir n'étant plus du ressort de seul Dieu mais aussi, comme expression d'une individualité en voie de constitution. Faute de pouvoir se réaliser dans la vie, on opte, selon une logique où le désespoir le plus sombre se mêle à la détermination la plus inflexible pour mener à bien sa mort et s'y affirmer, à défaut de le faire dans la vie. Que cette forme d'affirmation de soi se trouve liée à la manipulation la plus patente de la part des groupes ou des institutions (Bassidje en Iran), ne change rien à la situation: si le jeune est manipulé, c'est qu'il se laisse manipuler, qu'il est manipulable dans une conjoncture où la mort résout, à ses yeux, une situation inextricable. Dans le cas iranien, dans les dernières années de la guerre, cette forme de manipulation se révéla de plus en plus inopérante, tout simplement parce que les jeunes ne portaient plus en eux le désespoir révolutionnaire; dès lors, c'est en ayant recours à une logique purement coercitive et à des incitations financières que l'Etat islamiste tenta de les envoyer sur le front. Autant dire que la manipulation par des groupuscules ou des appareils étatiques n'explique pas à elle seule le mode d'action des jeunes et qu'il faut se placer dans leur subjectivité pour rehausser à la transparence leur mode d'action suicidaire.

Dans le déploiement même de l'activisme religieux où le sujet se constitue comme " individu dans le sacré " on assiste à des polarisations troublantes : tout ce qui était mis au service de la vie se trouve, passé un certain seuil d'implication de l'ego, converti en passion mortifère. A la vie collective se substitue la mort collective, à l'orgasme sexuel, l'extase mortifère, à la prise en charge de soi dans l'activisme au service d'un nouvel ordre à bâtir, la vision eschatologique d'une nouvelle société dans la mort, à la subordination de la mort à l'édification d'un idéal ici-bas, l'érection d'une forme de nécro-communauté qui devient la finalité suprême de l'action, toutes les autres considérations se trouvant marginalisées par cette véritable passion pour la mort qui caractérise une minorité des Bassidji où naît une " mystique mortifère " et qui vit littéralement en instance de mort, à la seule fin de mourir, transis par cette mort à venir, et, qui seule, donne sens à leur vie désertée par le sens. En d'autres termes, l'implication de ces jeunes " quasi-individus " dans un ensemble où ils parviennent à se soustraire mentalement aux aléas de la vie quotidienne leur ouvre une perspective imaginaire où ils " se réalisent " entièrement à l'abri du réel, dans un monde où le réel devient futile et où le combat contre la vie s'intériorise à tel point qu'ils cherchent, par tous les moyens, à mourir. Cette mort peut contribuer à l'indistinction de soi et d'autrui puisqu'il s'agit d'une mort béatifique qui ne saurait que faire du bien aux amis. Quant aux ennemis, ils font désormais partie d'une réalité qu'il faut briser au nom du primat 
de la mort. A mesure que ces jeunes plongent dans l'univers onirique d'une religiosité mortifère, la perspective du paradis, ou d'un après-vie, paré des attraits d'un monde d'espérances conjugué au futur s'estompe. Il ne s'agit plus de bâtir ce monde-là, mais, de mourir, et dans la mort, éprouver une extase sui generis, qui devient principe de motivation. Celle-ci se marie parfaitement avec une logique de désespoir généralisé : ce monde, le monde, le monde de la vie, ne sont pas les lieux de l'éclosion du sens. Il faut les supprimer et ce faisant, mettre fin à tous les modes d'expression de la vie, celle-ci étant coupable de se compromettre avec le réel.

La multiplication des formes de religiosité activiste et mortifère dans des situations extrêmes où l'horizon d'espérance est plus ou moins bouché (ou perçu tel par les jeunes acteurs) montre que la spécificité de tel type de religion (le chiisme, par exemple) n'est pas, en l'occurrence, une explication suffisante. La créativité juvénile et les groupes radicalisés engendrent une " chiisation "implicite à base d'un bricolage du religieux, qui peut aussi bien se réclamer du sunnisme dans le cas de l'islam (les jeunes Palestiniens sunnites du Hamas). L'enjeu essentiel est une modernité qui se donne sous une forme irrésistible dans les rêves, mais devient inaccessible dans la réalité quotidienne, là où précisément les industries culturelles répandent au niveau planétaire une image de l'ego iréniquement moderne, se réalisant dans une consommation euphorique, au sein d'un global village sans entraves. La déstructuration des formes traditionnelles et communautaires de gestion du sens libèrent un " quasi-individu " qui s'identifie aisément à cette modernité onirique, hors de sa portée. Dès lors, l'ego ne parvient à se réaliser que dans la mort, lorsqu'il est frustré des exigences les plus élémentaires de la modernité. La tentation est alors grande qu'il se dote d'une religiosité mortifère qui est le seul lieu où s'exerce sa créativité, faute de pouvoir se moderniser dans la vie. L'entreprise passe de l'état de projet à celui de réalité, là où des Etats (l'Iran) ou des groupes politiques apportent leur soutien à l'individu en désarroi, pour qui la quête de la mort est synonyme de salut. Notes.

* Farhad Khoroskhavar est maître de conférences à l'Ecole des hautes études en sciences sociales.

\section{NOTES}

1. Cf. dans L'Utopie Sacrifiée, op. cit., la section sur Khomeyni.

2. Cf. Bernard Hourcade, "Vaqf et Modernité en Iran : les agro-business de l'Astân-e qods de Mashad ", op. cit.

3. Cf. Ali Farâsati, " Kizéche hâyé sé sâlé gozachtéyé irân, marhaléyé boloughé mobârézâté mardomi " (en persan), [Les mouvements des trois dernières années en Iran, l'étape de maturité des luttes populaires] in Navidé Moghâvémat, $n^{\circ} 7$, printemps 1996, Paris.

4. Cf. Farhad Khosrokhavar, " Les intellectuels post-islamistes en Iran " in Awal, $\mathrm{n}^{\circ} 11$, 1994 ; cf. le dossier constitué par Nouchine Yavari-d'Hellencourt et Sétoreh Ghaffari- 
Farhangi, " Médias d'Iran et d'Asie Centrale " in Cemoti, n² 20, 1995 ; le dossier constitué par Fereydoun Khavand " L'Iran, quel avenir pour la République Islamique ? " in Trimestre du monde, 1er trimestre 1996.

5. Cf. Michel Wieviorka, Sociétés et terrorisme, Fayard, Paris, 1988 ; Face au terrorisme, Liana Levi, Paris, 1995.

6. On prend comme modèle des figures mineures d'autres jeunes ou des religieux ayant lutté contre les Britanniques et les Juifs de Palestine avant l'instauration de l'Etat d'Israël (Azzedine el-Qassem par exemple).

7. Cf. Abdulaziz A.Sachedina, Islamic Messianism. The Idea of the Mahdi in Twelver Shi'ism, Albany, Suny Press, 1981 ; Hamid Enayat, Modern Islamic Political Thought. The Response of the Shi'i Muslims to the Twentieth Century, Austin, University of Texas Press, 1982.

8. Le taux de croissance industrielle sous le régime du chah était élevé, même s'il y avait une grande disparité dans les formes d'industrialisation et une grande injustice dans la redistribution des fruits de la croissance. Cf. Jahangir Amuzegar, " The Iranian Economy before and after the Revolution ", Middle East Journal, 46 (3), 1992. Pour la période postrévolutionnaire, cf. Kaveh Ehsani, " Tilt but don't spill, Iran's Development and Reconstruction Dilemma ", Middle East Report, nov.-dec. 1994.

\section{INDEX}

Mots-clés : enfants, forces paramilitaires, kamikaze (missions suicide), conflits internationaux Index géographique : Iran

Thèmes : Bassidje, Pasdaran 